\title{
Un caso di malattia infiammatoria acuta intestinale da etilismo che mima una sindrome da carcinoide
}

\author{
Pietro Dattolo $^{1}$, Piercarlo Ballo², Stefano Michelassi ${ }^{1}$, Giulia Sansavini ${ }^{1}$, Giuseppe Ferro ${ }^{1}$, \\ Francesca Fusco ${ }^{3}$, Alam Mehmetaj ${ }^{1}$, Giuseppe Mangialavori ${ }^{2}$, Leandro Chiodi², \\ Francesco Pizzarelli ${ }^{1}$
}

\author{
${ }^{1}$ Nefrologia e Dialisi, Ospedale S.M. Annunziata, Firenze \\ ${ }^{2}$ Cardiologia, Ospedale S.M. Annunziata, Firenze \\ ${ }^{3}$ Radiologia, Ospedale S.M. Annunziata, Firenze
}

\begin{abstract}
ACUTe inflammatory Bowel
Abstract. We describe a case of acute inflammatory bowel disease complicating chronic alcoholism and mimicking a carcinoid syndrome, developed in a 65-year-old woman. She was admitted to our hospital because of refractory diarrhea with severe hypokalemia $(1.7 \mathrm{mEq} / \mathrm{L})$. Abdominal multi-detector computed tomography, performed before and after intravenous contrast media administration (with double arterial and portal phase), showed diffuse inflammation of the entire colon with severe wall thickening and mural stratification, suggesting a non-specific inflammatory bowel disease. No involvement of the small bowel was found, and there were no findings suggestive of carcinoid tumor or specific inflammatory bowel diseases. Colonoscopy also showed diffuse edema and hyperemia of colic mucosa with no evidence of ulcerative lesions. Multiple colonic biopsies confirmed a non-specific inflammatory bowel disease with no evidence of carcinoid cells. In conclusion, this report suggests that chronic alcohol abuse may lead to acute reversible inflammatory bowel disease, refractory diarrhea and severe hypokalemia, with clinical and laboratory features that can mimic those of a carcinoid syndrome.
\end{abstract}

Key words: IBD, Alcohol, Hypokalemia, Complicating chronic, Alcoholism and mimicking, Carcinoid syndrome

Conflict of interest: None.

Ricevuto: 21 Giugno 2012; Accettato: 28 Novembre 2012

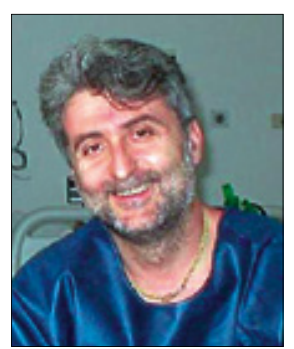

Una donna di 65 anni, obesa, con storia di abuso cronico di alcol e con recente frattura lombare traumatica è stata trasferita presso il nostro Ospedale da un Centro di riabilitazione per l'insorgenza di diarrea refrattaria ed ipokaliemia severa $(1.7 \mathrm{mEq} / \mathrm{L})$. La paziente era in trattamento con acido folico, sulpiride, ramipril, spironolattone, cloruro di Pietro Dattolo potassio, delorazepam e nadroparina. All'ingresso in reparto si presentava in scadenti condizioni generali, disorientata nel tempo e nello spazio, soporosa ed irritabile; la pressione arteriosa era 160/100 mmHg e la frequenza cardiaca 96 bpm. Non erano presenti segni obiettivi di rilievo all'esame toracico e cardiovascolare. L'ECG mostrava tachicardia sinusale mentre l'ecocardiogramma non evidenziava alterazioni patologiche. All'emogasanalisi arteriosa era presente severa alcalosi metabolica e gli esami ematici mostravano anemia microcitica (Hb 9.8 g/dL, MCV 63.5 fl, MCH 20.7 pg), ipoproteinemia $(4.4 \mathrm{~g} / \mathrm{dL})$ ed incremento dei livelli sierici di gamma-GT (94 IU/L), ammonio (71 $\mu \mathrm{g} / \mathrm{dL})$, NT-proBNP (1151 pg/mL) e PCR $(2.58 \mathrm{mg} / \mathrm{dL})$. A causa dell'elevato rischio aritmogeno legato alla severa deplezione di potassio, la paziente è stata trasferita presso l'Unità coronaria e trattata con potassio ad alte dosi (fino a $160 \mathrm{mEq} / \mathrm{die}$ ), ed impostata terapia antibiotica con rifamixina.

I test microbiologici sulle feci erano tutti negativi, inclusa la ricerca del Clostridium difficile e della sua tossina. Inoltre, le indagini laboratoristiche effettuate sul sangue e sulle urine per escludere cause tumorali, infettive, endocrinologiche e reumatologiche risultarono, ad eccezione del rilievo di un incremento dell' acido 5-idrossindolacetico urinario $(12.3 \mathrm{mg} / 24$ h, v.n. $<9.0 \mathrm{mg} / 24 \mathrm{~h}$ ), del cortisolo urinario (810 microng/24 h, v.n. $<100$ microng/24 h) e del CA125 sierico (150 IU/mL, n.v. $<35 \mathrm{IU} / \mathrm{mL}$ ). 


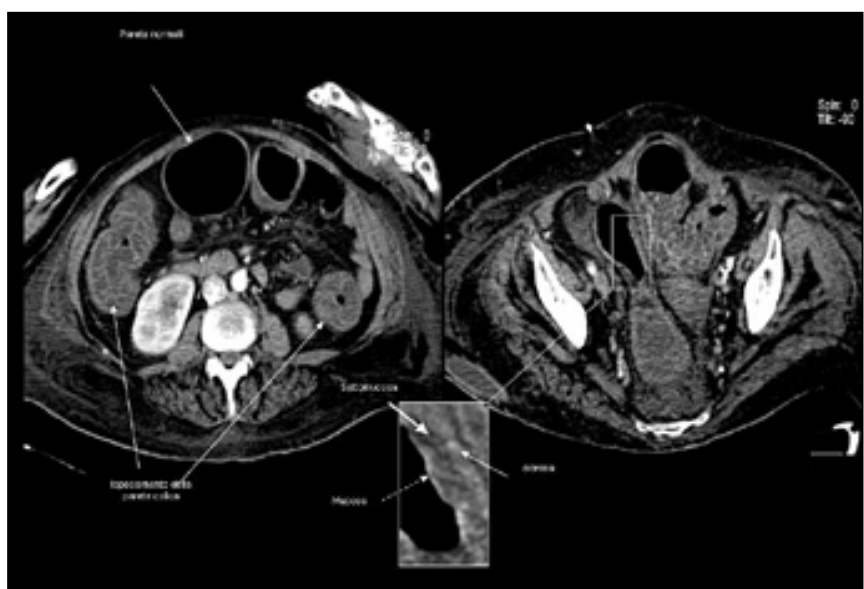

Fig. 1 - Immagini TC che mostrano una severa infiammazione del colon. È evidente un marcato ispessimento della parete colica con aspetto trilaminare per incremento dello spessore della sierosa, iperemia della mucosa e attenuazione dello strato submucosale interposto, dovuti ad edema acuto della parete. Questi aspetti sono suggestivi per malattia infiammatoria intestinale acuta aspecifica.

Nonostante il trattamento, la diarrea e la severa ipokaliemia persistevano durante i giorni seguenti. Al sesto giorno di ricovero i livelli di potassio erano ancora intorno a $2.2 \mathrm{mEq} / \mathrm{L}$. Erano inoltre presenti nausea, vomito, dolore addominale e ipertensione (fino a $167 / 104 \mathrm{mmHg}$ ) con saltuarie crisi ipotensive (fino a $85 / 50 \mathrm{mmHg}$ ), che hanno indirizzato verso un sospetto di sindrome da carcinoide.

Un'ecografia addominale mostrava un'architettura epatica disomogenea in assenza di altre anomalie significative, e una TC dell'addome multi-slide, eseguita prima e dopo somministrazione endovenosa di mezzo di contrasto con doppia fase arteriosa e portale, mostrava un'infiammazione diffusa di tutto il colon con severo ispessimento della parete e stratificazione murale; tale reperto era suggestivo per malattia infiammatoria intestinale aspecifica (Fig. 1). Non era documentabile invece alcun coinvolgimento dell'intestino tenue e non erano presenti segni suggestivi per tumore carcinoide o per malattie infiammatorie croniche intestinali specifiche (in particolare non erano presenti, strie di grasso, ulcere mucose o tumefazione dei linfonodi extramurali) con prominenza dei vasa recta a livello mesenteriale. Anche la colonscopia mostrava edema diffuso ed iperemia della mucosa colica, in assenza di lesioni ulcerative; biopsie multiple della mucosa colica hanno confermato la presenza di una malattia infiammatoria intestinale aspecifica, senza evidenza di cellule da carcinoide.

Nei giorni successivi la diarrea è andata incontro a una lenta remissione e le condizioni cliniche della paziente sono progressivamente migliorate. Al ventunesimo giorno di ricovero il valore di potassio era $2.7 \mathrm{mEq} / \mathrm{L}$.

La paziente è stata in seguito trasferita presso una Casa di cura dove ha iniziato un programma educazionale per ridurre il consumo alcolico. I valori di potassio sono rientrati nel range di normalità dopo diverse settimane. A un anno di follow-up dopo la sospensione dell'abuso alcolico le condizioni generali della paziente erano buone: la paziente non ha più presentato diarrea $\mathrm{e} i$ valori di potassio si sono mantenuti intorno a $4.5 \mathrm{mEq} / \mathrm{L}$.

\section{Discussione}

Con questo case report descriviamo il caso di una donna alcolista che ha sviluppato severa ipokaliemia secondaria a diarrea refrattaria con evidenza strumentale di marcata infiammazione della parete colica. Tale condizione suggerisce l'interessante possibilità di una forma di malattia infiammatoria acuta intestinale alcol-correlata.

A nostra conoscenza, non sono riportati in letteratura precedenti casi di malattia infiammatoria intestinale associata all'utilizzo cronico di alcol. È noto che l'esposizione cronica all'alcol è responsabile di numerosi effetti dannosi sulla mucosa intestinale, dipendenti dalla complessa interazione tra l'incremento dello stress ossidativo, l'iperpermeabilità, la neuropatia alcolica e il dismetabolismo che col tempo favoriscono e sostengono l'infiammazione locale $(1,2)$. L'alcol può inoltre giocare un ruolo favorente l'aggravamento della malattia nei pazienti già affetti da malattie infiammatorie intestinali (3).

Da un altro punto di vista, è importante considerare che la sindrome da carcinoide - condizione caratterizzata da una serie di segni e sintomi dovuti all'incremento dei livelli ematici di serotonina prodotta da cellule neoplastiche neuroendocrine e presenti nel $10 \%$ dei pazienti con tumori carcinoidi - è caratterizzata da una combinazione variabile di sintomi che possono includere diarrea refrattaria, flushing, squilibri elettrolitici, nausea, vomito, crampi addominali, crisi ipo- o ipertensive, ronchi sibilanti all'esame obiettivo del torace e talvolta scompenso cardiaco destro. Anche disordini del comportamento come irritabilità e mancanza di controllo degli impulsi sono relativamente comuni nei pazienti con sindrome da carcinoide, e si manifestano conseguentemente ad anomalie che si instaurano a carico del sistema serotoninergico centrale (4). È inoltre da notare che diarrea e flushing - i due sintomi più tipici della sindrome da carcinoide - sono entrambi presenti soltanto nel $58 \%$ dei casi, e possono essere entrambi assenti nel $22 \%$ dei casi $(5,6)$.

La diagnosi si basa sul quadro clinico, su caratteristici aspetti laboratoristici, come l'aumento dell'escrezione urinaria di acido 5-idrossindolacetico, su caratteristiche alterazioni morfologiche e funzionali rilevate con metodiche di imaging come l'ecografia standard, la tomografia computerizzata e la scintigrafia, ed il rilievo di cellule tumorali neuroendocrine alla biopsia della mucosa intestinale. Agli esami di laboratorio possono inoltre comparire un aumento del cortisolo urinario e un aumento dei livelli ematici di alcuni marcatori tumorali, come il CA125 $(7,8)$.

È interessante notare che la presentazione clinica della paziente ed il quadro laboratoristico all'esordio mimavano le caratteristiche di una sindrome da carcinoide. La paziente si presentava infatti con diarrea refrattaria, ipokaliemia severa, dolore addominale, alternanza di ipertensione seguita da crisi ipotensive e disordini del comportamento e mancanza 
di controllo dell'impulsività. Gli esami di laboratorio mostravano un incremento dei livelli ematici del CA125 e dei livelli urinari di acido 5-idrossindolacetico e del cortisolo. Questi dati erano concordanti con l'ipotesi di sindrome da carcinoide, ma biopsie intestinali multiple non confermavano la diagnosi. Nonostante la mancata esecuzione dell'esame scintigrafico per rifiuto della paziente non abbia permesso di escludere definitivamente la presenza di una sindrome da carcinoide, è possibile considerare che la diarrea, l'ipokaliemia ed il dolore addominale possano essere stati verosimilmente causati dall'abuso cronico di alcol, in relazione al suo effetto dannoso sulla mucosa intestinale. L'assunzione cronica di alcol è inoltre tipicamente associata ad elevati valori pressori (9) e comunemente vengono osservate nei pazienti alcolisti ospedalizzati anomalie nella regolazione della pressione arteriosa, che si manifestano con crisi ipotensive durante sindrome da astinenza alcolica conseguenti a una ridotta sensibilità dei barocettori (10). I disordini del comportamento con anomalie nel controllo dell'impulsività possono essere favorite dall'assunzione cronica di alcol, in rapporto agli effetti che questa sostanza ha sul sistema nervoso centrale $(11,12)$. Inoltre, come risultato della sindrome da malassorbimento, l'esposizione cronica all'alcol è spesso associata a un incremento dell'escrezione urinaria dei metaboliti del triptofano e della serotonina, come l'acido 5-idrossindolacetico (13), mentre la disregolazione alcolcorrelata dell'asse ipotalamo-ipofisi-surrene può condurre a un incremento del cortisolo urinario (14). Infine, anche il miglioramento della sintomatologia nella nostra paziente e la normalizzazione dei livelli ematici di potassio ad un anno di follow-up dopo la sospensione del consumo alcolico, supportano l'ipotesi di una malattia infiammatoria intestinale acuta alcol-correlata.

Concludendo questo report suggerisce che l'abuso cronico di alcol possa condurre ad una malattia infiammatoria intestinale acuta reversibile con diarrea refrattaria e severa ipokaliemia, le cui caratteristiche cliniche ed i rilievi laboratoristici possono mimare una sindrome da carcinoide.

\section{Riassunto}

Descriviamo il caso di malattia infiammatoria acuta dell'intestino insorta in una donna di 65 anni, etilista e obesa. La paziente è stata ricoverata per diarrea refrattaria ed ipopotassiemia severa $(1.7 \mathrm{mEq} / \mathrm{L})$. Una TC dell'addome, con mdc (con doppia fase arteriosa e portale), mostrava un'infiammazione diffusa di tutto il colon con severo ispessimento della parete e stratificazione murale, reperto suggestivo per malattia infiammatoria intestinale aspecifica. Non era documentabile alcun coinvolgimento dell'intestino tenue e non erano presenti segni suggestivi per carcinoide o per malattie infiammatorie croniche intestinali specifiche. La colonscopia mostrava edema diffuso ed iperemia della mucosa colica, in assenza di lesioni ulcerative; biopsie multiple della mucosa colica hanno confermato la presenza di una malattia infiammatoria intestinale aspecifica, senza evidenza di cellule da carcinoide. Concludendo, questo report suggerisce come l'abuso cronico di alcol possa condurre a una malattia infiammatoria intestinale acuta reversibile con diarrea refrattaria e severa ipopotassiemia, le cui caratteristiche cliniche ed i rilievi laboratoristici possono mimare una sindrome da carcinoide.

Parole chiave: Malattia infiammatoria acuta dell'intestino, Carcinoide, Alcolismo, Ipopotassiermia

Dichiarazione di conflitto di interessi: Gli Autori dichiarano di non avere conflitto di interessi.

Indirizzo degli Autori:

Dr. Pietro C. Dattolo

UO Nefrologia e Dialisi

Ospedale S M Annunziata

Via dell'Antella 58

50011 Antella (FI)

pcdattolo@gmail.com

\section{Bibliografia}

1. Keshavarzian A, Farhadi A, Forsyth CB, et al. Evidence that chronic alcohol exposure promotes intestinal oxidative stress, intestinal hyperpermeability and endotoxemia prior to development of alcoholic steatohepatitis in rats. J Hepatol 2009; 50: 538-47.

2. Mutlu E, Keshavarzian A, Engen P, Forsyth CB, Sikaroodi M, Gillevet P. Intestinal dysbiosis: a possible mechanism of alcohol-induced endotoxemia and alcoholic steatohepatitis in rats. Alcohol Clin Exp Res 2009; 33: 1836-46.

3. Swanson GR, Sedghi S, Farhadi A, Keshavarzian A. Pattern of alcohol consumption and its effect on gastrointestinal symptoms in inflammatory bowel disease. Alcohol 2010; 44: $223-8$
4. Russo S, Boon JC, Kema IP, Willemse PH, den Boer JA, Korf J, de Vries EG. Patients with carcinoid syndrome exhibit symptoms of aggressive impulse dysregulation. Psychosom Med 2004; 66: 422-5.

5. Vinik AI, Feliberti E, Perry R, Nakave A. (2008). Diffuse Hormonal Systems and Endocrine Tumor Syndromes. Retrieved from: http://www.endotext.org/guthormones/index.htm/

6. 6. Van der Lely AJ, de Herder WW. Carcinoid syndrome: diagnosis and medical management. Arq Bras Endocrinol Metabol 2005; 49: 850-60.

7. Tanke MA, Kema IP, Dijck-Brouwer J, Doornbos B, De Vries EG, Korf J. Low plasma tryptophan in carcinoid patients is associated with increased urinary cortisol excretion. Psychoneuroendocrinology 2008; 33: 1297-301.

8. Portale TR, Mosca F, Minona E, Trovato MA, Gangemi P, 
Bordonaro R, Puleo S. Gastrointestinal carcinoid tumor and chylous ascites, a rare association with a poor prognosis. A case report. Tumori 2008; 94: 419-21.

9. Marmot MG, Elliott P, Shipley MJ, et al. Alcohol and blood pressure: the INTERSALT study. BMJ 1994; 308:1263-7.

10. Bär KJ, Boettger MK, Boettger S, et al. Reduced baroreflex sensitivity in acute alcohol withdrawal syndrome and in abstained alcoholics. Drug Alcohol Depend 2006; 85: 66-74.

11. Rogers RD, Moeller FG, Swann AC, Clark L. Recent research on impulsivity in individuals with drug use and mental health disorders: implications for alcoholism. Alcohol Clin Exp Res 2010; 34:1319-33.

12. 1Schellekens AF, de Bruijn ER, van Lankveld CA, et al. Alcohol dependence and anxiety increase error-related brain activity. Addiction 2010; 105: 1928-34.

13. Das SK, Dhanya L, Vasudevan DM. Biomarkers of alcoholism: an updated review. Scand J Clin Lab Invest 2008; 68: 81-92.

14. Thayer JF, Hall M, Sollers JJ 3rd, Fischer JE. Alcohol use, urinary cortisol, and heart rate variability in apparently healthy men: Evidence for impaired inhibitory control of the HPA axis in heavy drinkers. Int J Psychophysiol 2006; 59: 244-50.

\section{TEST DI VERIFICA}

\section{1) Il dosaggio nelle feci della calprotectina è utile:}

a) Come indice di uno stato infiammatorio intestinale di tipo organico, in particolare per le MICI (Malattie Croniche Infiammatorie Intestinali).

b) Per il monitoraggio dello stato infiammatorio intestinale

c) Predittività delle recidive

d) Tutte le condizioni sopra indicate

e) Nessuna delle condizioni indicate

2) Il consumo di alcol nelle malattie infiammatorie croniche dell'intestino:

a) Aumenta il rischio di recidive fino a tre volte

b) È indifferente sulla insorgenza di recidive

c) Diminuisce il rischio di recidive fino a tre volte

3) Qual è l'elemento morfologico caratteristico che ci permette di diagnosticare una colite infettiva:

a) Normale architettura degli elementi ghiandolari

b) Aumento cellularità lamina propria

c) Infiltrato neutrofilo nelle fasi iniziali

d) Infiammazione discontinua e focale criptite

e) Tutti i precedenti

f) Nessuno dei precedenti

Le risposte corrette alle domande sono pubblicate su questo numero del Giornale di Tecniche Nefrologiche \& Dialitiche Vol. 25, no. 1, pag. 52. 